\title{
Focused Ion Beam (FIB) Preparation and Electron Microscopy Analysis of Individual Microbolometer Pixels
}

\author{
M. Olszta*, J. Dougherty****, M. Horn*, and E. C. Dickey* \\ *The Materials Research Institute (MRI), Pennsylvania State University, 101 Materials Research \\ Institute Building, University Park, PA 16802, United States \\ **Electro-Optics Center (EOC), Pennsylvania State University, 222 North Pointe Blvd, \\ Freeport, PA 16229, United States
}

In an ever turbulent political and military landscape, it becomes necessary to develop detection technologies which can cover a wide-range of applications. One such technology, un-cooled infrared (IR) detectors, is being utilized in a variety of national security applications, ranging from night-vision cameras, to missile tracking systems to screening detectors for concealed weaponry. Previous generations of IR detectors required large cooling apparatus in order to maintain sub-zero temperatures that would ensure proper detection. Microbolometer technology, with a free-standing design (Figure 1A, B), is one of the leading candidates to replace the large, cryogenically cooled systems. The free-standing design is comprised of a 1-2 $\mu \mathrm{m}$ thick pixel (Figure 2) which is suspended above a reflector by two metallic legs (Figure 1B). IR detection is measured by a small change in resistance in the $\sim 125 \mathrm{~nm}$ thin vanadium oxide $\left(\mathrm{VO}_{\mathrm{x}}\right)$ film, and thus the chemical composition, microstructure, and continuity of this layer is quite important. Due to highly involved and intricate processing techniques, the morphology of each individual pixel is also important to the efficacy of finished microbolometer devices. The spatial geometry of each pixel requires that (each pixel $\sim 50 \times 50 \mu \mathrm{m}^{2}$ ) the analysis of materials properties of individual pixels as related to processing and failure must be performed by transmission electron microscopy (TEM). Because these detectors are comprised of thousands of pixels in a space no larger than a square inch, conventional sample preparation is not suitable.

Analysis of individual pixels (each no larger than $50 \times 50 \mu \mathrm{m}^{2}$ and 1-2 $\mu \mathrm{m}$ thick (Figure 1B, 2B)) requires that focused ion beam (FIB) be used for preparing site specific TEM samples. Using FIB, a single pixel was removed from the microbolometer device using a tungsten (W) microprobe and subsequently attached to a copper $(\mathrm{Cu})$ TEM grid. The pixel was then milled to electron transparency using decreasingly smaller ion currents $(20 \mathrm{nA}-10 \mathrm{pA})$. The contrast between the vanadium and silicon oxides provided a watermark for milling (Figure 2A) such that caution could be taken not to mill too close to the $\mathrm{VO}_{\mathrm{x}}$ layer. Samples were subsequently analyzed in the TEM using bright field (BF) (Figure 2B), selected area diffraction (SAD), electron energy loss (EELS) (Figure 3), electron diffraction (ED), energy dispersive spectroscopy (EDS) and scanning transmission electron microscopy (STEM). Using the FIB to prepare site specific samples allows for analysis of each material layer of an individual pixel (Figure 3).

We have demonstrated that site specific sample preparation using the FIB, in combination with analytical TEM, is a powerful, and necessary, tool in the analysis of next generation IR detectors. 

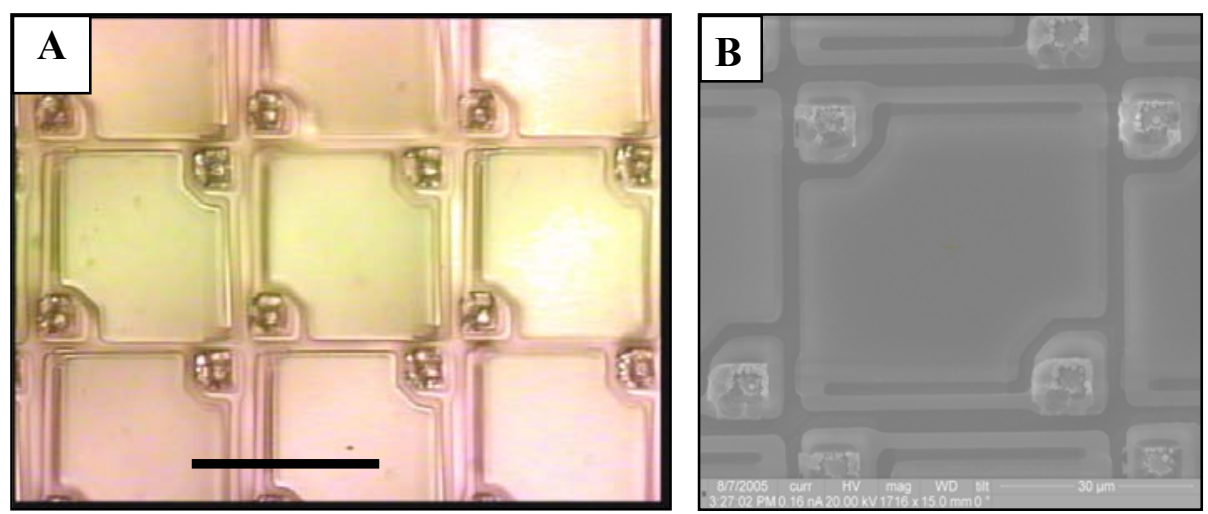

Figure 1: Optical and electron micrographs illustrating the design of individual microbolometer pixels. A. Optical micrograph of microbolometer pixel array. Bar $=50 \mu \mathrm{m}$. B. Scanning electron micrograph of an individual pixel. $\mathrm{Bar}=30 \mu \mathrm{m}$.
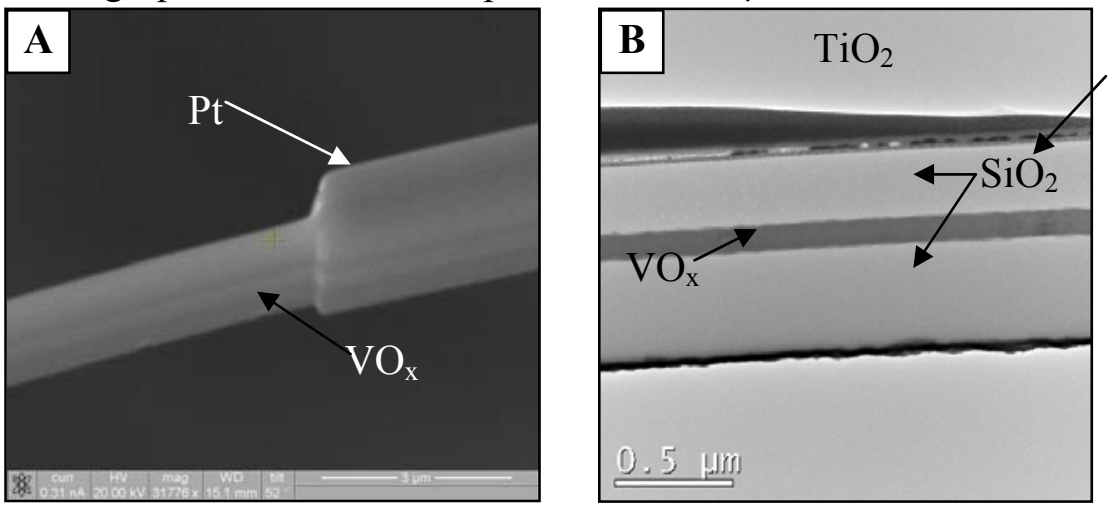

Figure 2: Electron micrographs illustrating the thin film morphology of an individual pixel. A. Secondary electron image showing the contrast change in the $\mathrm{VO}_{\mathrm{x}}$ layer which is used as a watermark during milling in order to avoid over-milling the pixel. Bar $=3 \mu \mathrm{m}$. B. Free standing pixels are $\sim 1-2 \mu \mathrm{m}$ thick, with four layers of different materials. Bar $=0.5 \mu \mathrm{m}$.

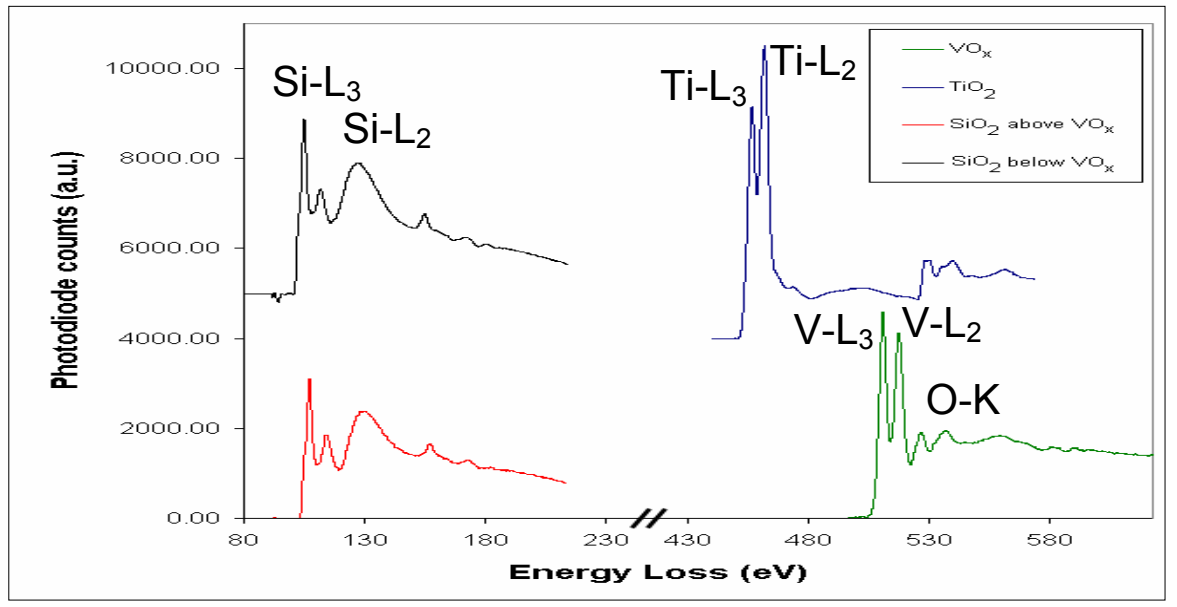

Figure 3: EELS analysis of individual layers in a microbolometer pixel. 